\title{
A review of the solar results from CORONAS-F satellite
}

\author{
V.D.Kuznetsov ${ }^{1}$, Yu.E.Charikov ${ }^{2}$, Yu.D.Kotov ${ }^{3}$, S.N.Kuznetsov ${ }^{4}$, \\ E.P.Mazets ${ }^{2}$, \\ A.A.Nusinov ${ }^{5}$, V.M.Pankov ${ }^{6}$, I.I.Sobelman ${ }^{7}$, J.Sylwester ${ }^{8}$ \\ ${ }^{1}$ Institute of Terrestrial Magnetism, Ionosphere, and Radio Wave Propagation, Russian \\ Academy of Sciences, 142190, Moscow Region, Troitsk, IZMIRAN, Russia, e-mail: \\ kvd@izmiran.ru \\ ${ }^{2}$ Ioffe Institute of Physics and Technology, Russian Academy of Sciences, 194021, St- \\ Petersburg,Politechnicheskaya str.26, Russia; e-mail: yuri.charikov@mail.ioffe.ru; \\ mazets@mail.ioffe.ru \\ ${ }^{3}$ Moscow Physical Engineering Institute,115409, Koshirskoe shosse 31, Moscow, Russia, e-mail: \\ kotov@mephi.ru \\ ${ }^{4}$ Research Institute of Nuclear Physics, Moscow State University, 119899 Vorob'evi Gory, \\ Moscow, Russia, e-mail: kuznets@srd.sinp.msu.ru \\ ${ }^{5}$ Institute of Applied Geophysics, Russian Committee for Hydrometeorology, 129128, Moscow, \\ Rostokinskaya str.9, Russia, e-mail: nusinov@mail.ru \\ ${ }^{6}$ Space Research Institute, Russian Academy of Sciences,117997, Moscow, Profsoyznaya \\ str.84/32, e-mail:vpan@iki.rssi.ru \\ ${ }^{7}$ Lebedev Physical Institute, 117997, Moscow, Leninsky prospekt 53, Russia, e-mail: \\ kuzin@sci.lebedev.ru \\ ${ }^{8}$ Space Research Centre, Polish Academy of Sciences, 51-622, Kopernika 11, Wroclaw, \\ e-mail:js@cbk.pan.wroc.pl
}

\begin{abstract}
The solar results from CORONAS-F satellite have been reviewed. The observations with the DIFOS multi-channel photometer in a broad spectral range from 350 to $1500 \mathrm{~nm}$ have allowed to determine the dependence of the relative amplitudes of p-modes of the global solar oscillations on the wavelength. The EUV observations in SPIRIT experiment have enabled the study of various manifestations of solar activity and high-temperature events on the Sun. The data from the flare instruments - gamma spectrometer HELICON, flare spectrometer IRIS, amplitude-time spectrometer AVS-F, and X-ray spectrometer RPS-1 have been used to analyze the hard emission from solar flares and to carry out the diagnostics of the solar flare plasma. The Solar Cosmic Ray Complex has investigated the solar flare effects in the Earth's environment. The UV emission variations during solar flares in the vicinity of the $120-\mathrm{nm}$ wavelengh have been recorded and the relative variation amplitude has been determined.
\end{abstract}

\section{Introduction}

The joint Russian-Ukrainian satellite CORONAS-F launched three years ago (on July $31,2001)$ continues observations of solar activity and solar-terrestrial coupling. The satellite is orbiting the Earth at a height of about $500 \mathrm{~km}$; the orbit inclination is $83 \mathrm{deg}$. The scientific payload of the satellite comprises 15 instruments, which enable observations of the Sun in the entire electromagnetic spectrum from optical radiation to gamma rays. 
Table 1. Measuring channels of the CORONAS-F instruments

\section{Helioseismology}

Spectrophotometer DIFOS

\begin{tabular}{|c|c|}
\hline Gamma Spectrometer HELIKON & $10 \mathrm{keV} \div 10 \mathrm{MeV}$ \\
\hline Flare Spectrometer IRIS & $2 \div 200 \mathrm{keV}$ \\
\hline X-ray Spectrometer RPS-1 & $2 \div 20 \mathrm{keV}$ \\
\hline Spectrophotometer DIOGENESS & $3 \div 7 \AA$ \\
\hline X-Ray Spectrometer RESIK & $1 \div 6 \AA$ \\
\hline Amplitude-Time Spectrometer AVS & $3 \div 30 \mathrm{keV} ; 0,1 \div 0,8 \mathrm{MeV} ; 2 \div 80 \mathrm{MeV}$ \\
\hline Solar Spectropolarimeter SPR-N & $20 \div 100 \mathrm{keV}$ \\
\hline $\begin{array}{l}\text { SPIRIT Experiment (Solar X-Ray Telescope SRT- } \\
\text { K, X-Ray Spectroheliograph RES-K) }\end{array}$ & $1,85 \div 335 \AA$ \\
\hline \multicolumn{2}{|l|}{ Ultra Violet Emission of the Sun } \\
\hline Solar UV Radiometer SUFR-Sp-K & $10 \div 1300 \AA$ \\
\hline Solar UV Spectrophotometer VUSS-L & $121.6 \mathrm{~nm} \pm 5 \mathrm{~nm}$ \\
\hline \multicolumn{2}{|l|}{ Solar Cosmic Rays } \\
\hline Gamma Emission Spectrometer SONG & $\begin{array}{c}\gamma 0,03 \div 100 \mathrm{MeV} ; \gamma 0,3 \div 20 \mathrm{MeV} ; n 20 \mathrm{MeV} ; \\
e 50 \mathrm{MeV} ; p 70 \mathrm{MeV}\end{array}$ \\
\hline Cosmic Rays Monitor MKL & $\gamma 1 \div 200 \mathrm{MeV} ;$ e $0,5 \div 12 \mathrm{MeV}$ \\
\hline Cosmic Emission Spectrometer SKI-3 & $1,5 \div 20 ; 4 \div 40 \mathrm{MeV} / \mathrm{n}$ \\
\hline
\end{tabular}

\section{Measuring ranges}

$350-1500 \mathrm{~nm}$

\section{Solar Flares and Imaging of the Sun}

\section{Spirit experiment}

Within the SPIRIT experiment, the Solar X-Ray Telescope SRT-K and X-Ray Spectroheliograph RES-K recorded the most intensive recent active events of October-November, 2003 (Fig.1) and coronal mass ejections at a speed of hundreds of $\mathrm{km} / \mathrm{s}$. The data obtained allow us to study the morphology and dynamics of the flares, mass ejections and the associated dimmings. It is found out that the dimmings observed were mainly due to the full or partial opening of the magnetic fields during coronal mass ejections and outflow of matter from large-scale magnetic features in the transition region and corona 


\section{October 2003}
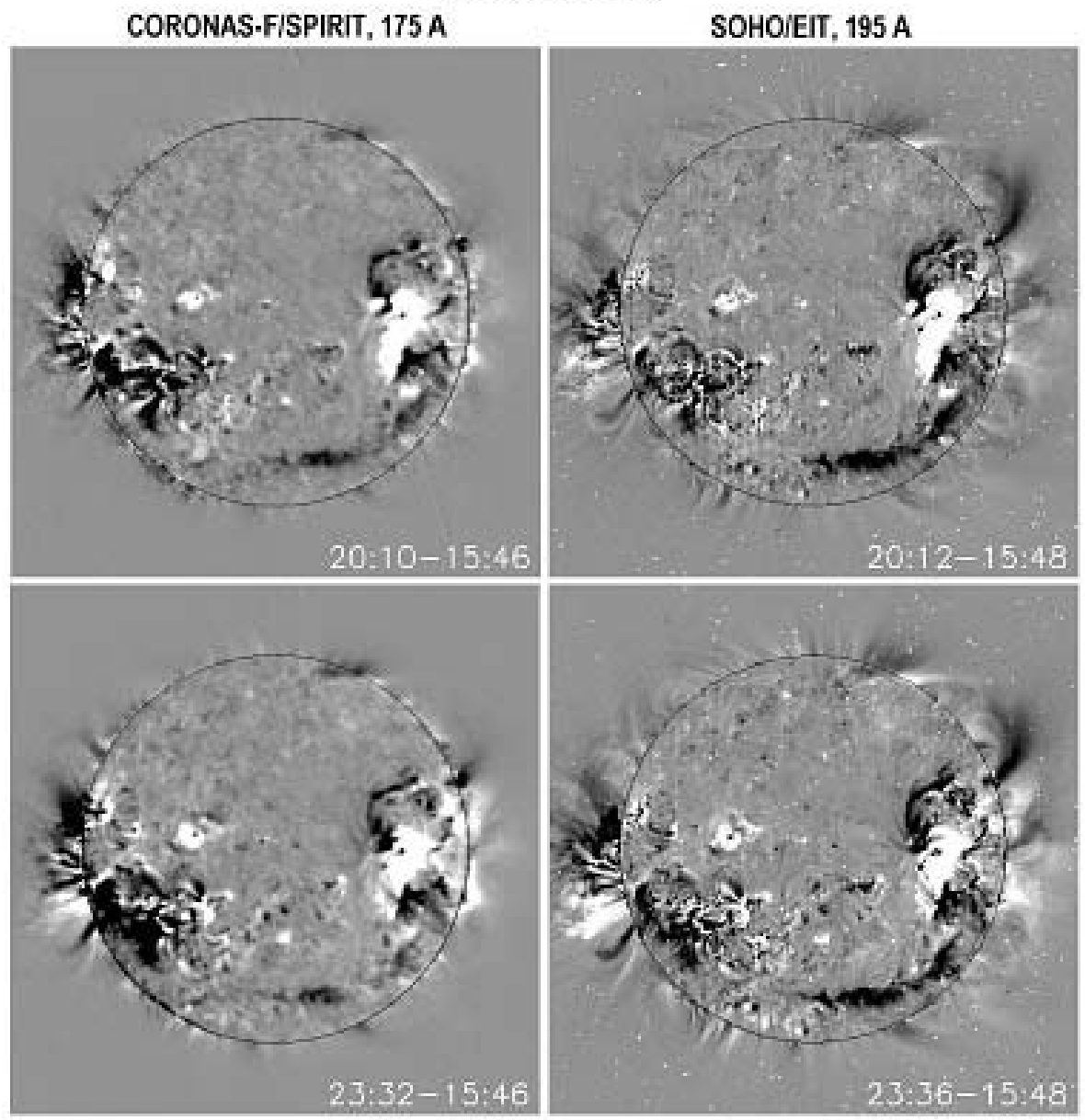

Figure 1. Powerful solar flares and ejections on 26 October 2003.

and, as a result, to the decrease of the emission measure. The global nature of the dimmings in that and other events implies that a significant part of the solar atmosphere was involved in the eruptive process. A homology of the eruptive events has been revealed; i.e., the pattern of the dimmings repeated in the main features from event to event; and each time, the coronal mass ejection involved approximately the same objects, which, apparently, could recover their magnetic field and brightness between the events.

Observations in the MgXII (8.42 A) resonance line using a new method of the full-Sun monochromatic imaging spectroscopy have revealed fast dynamic regions of hot plasma with the temperatures up to 10 million degrees $\mathrm{K}$ in the solar corona. They reflect fast processes in the flare arches and allow a deeper insight into the active solar phenomena that were not properly understood before. It is found out that such high-temperature plasma is present in the solar corona almost permanently. The phenomena recorded are characterized by a broad range of brightness (up to 4 orders) and large lifetime - from seconds to days. Various phases of these phenomena have been studied with a high temporal resolution $(50 \mathrm{~s})$. These observations of the dynamic solar plasma with a temperature of about 10 million deg.K allowed us to unify data on the "stationary" plasma (1-3 mln.deg K) and high-temperature transient and flare plasma (over 15 mln.deg 


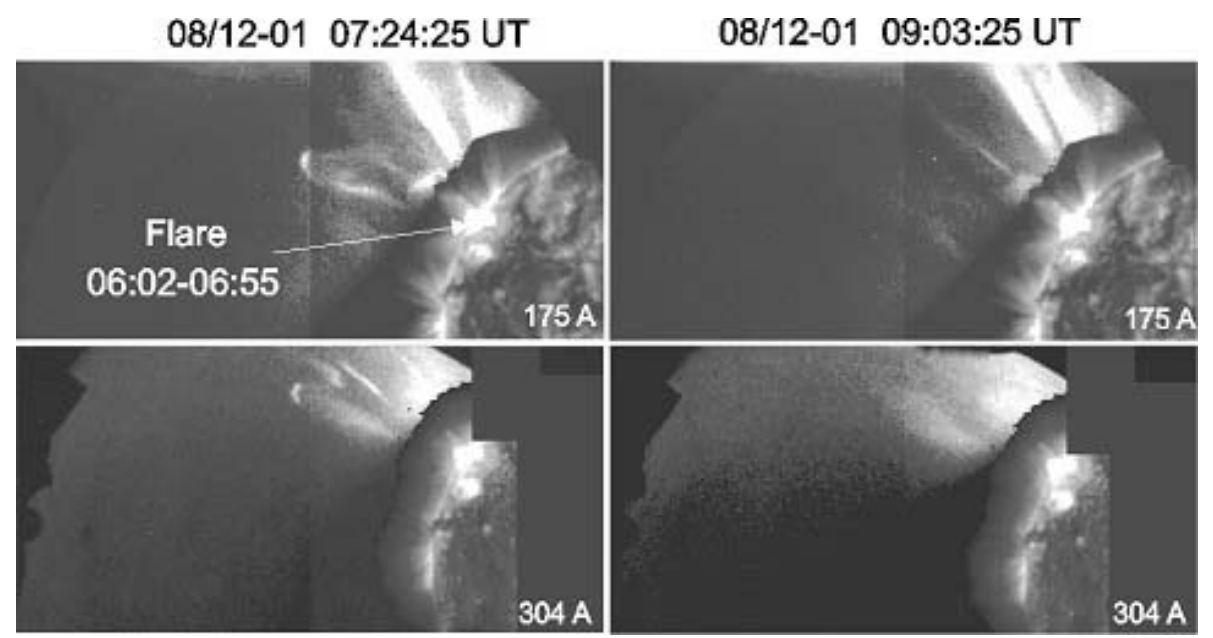

Figure 2. The stationary and disturbed solar corona observed by Hard X-ray Coronograph

K) provided by the space missions SOHO, TRACE, RHESSI, GOES, and CORONASF.

Simultaneous observations at two wavelengths (175 and 304 A) using a Hard X-ray Coronograph have enabled the study of the stationary and disturbed solar corona at 3 solar radii, as well as various active events, such as mass ejections, eruptive prominences, jets, etc (Fig.2).

More than 170 spectral lines of various chemical elements have been identified and analyzed in the coronal X-ray spectrum.

In the study of solar-terrestrial coupling, new experimental data have been obtained on variations of the density and composition of the Earth's atmosphere up to $500 \mathrm{~km}$ and their relation to solar activity. It is established how the penetration of solar X-rays in the range of 8.42, 175, and 304 A through the Earth's atmosphere depends on the minimum height of the beam over the Earth's surface.

\section{Solar flares}

A number of the CORONAS-F instruments are designed to measure the Solar Flare Emission in a broad energy range with a high temporal and spectral resolution. These instruments have provided new experimental data on the physical processes in solar flares, such as build-up and release of the magnetic energy, energy spectra, nuclear lines, gamma-radiation time profiles, X-ray dynamic spectra, profiles of various spectral lines of the flare plasma, polarization of the flare-generated X-rays, etc.

The sensitive X-Ray Spectrometer RPS-1 recorded pre-flare enhancements and minor $\mathrm{X}$-ray flares (importance $\mathrm{C}$ and $\mathrm{M}$ ) in the energy range of 3-30 keV. The spectrum rigidity of those events was determined in different phases of the flare. It was shown that the most rigid X-ray spectra were observed at the peak of the flare evolution (Fig.3).

The dependence of the X-ray background spectrum on solar activity (sunspot number) has been studied in the absence of flares (in the period of October 2003 characterized by dramatic variation of the solar activity level). At the sunspot minimum, the spectrum was soft and did not exceed 6-7 keV. At the sunspot maximum, the spectrum was hard and reached $20 \mathrm{keV}$, which was due to the appearance of very hot regions (AR 484 and AR 


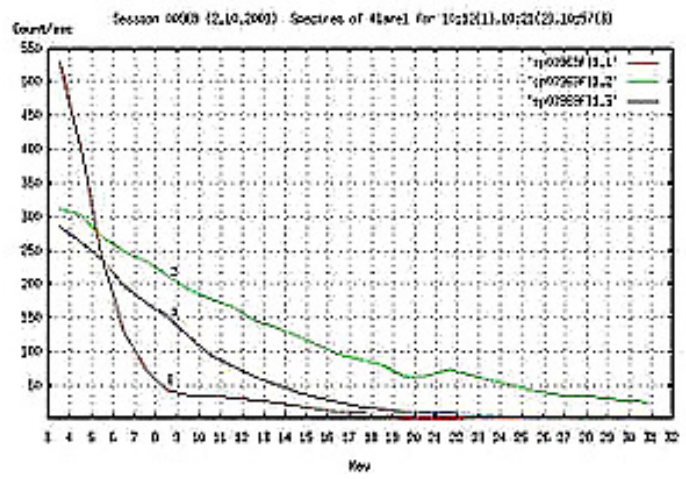

Figure 3. Spectra of the class M flare emission: 1 - the onset of the flare, 2 - the maximum of the flare, 3 - the end of the flare
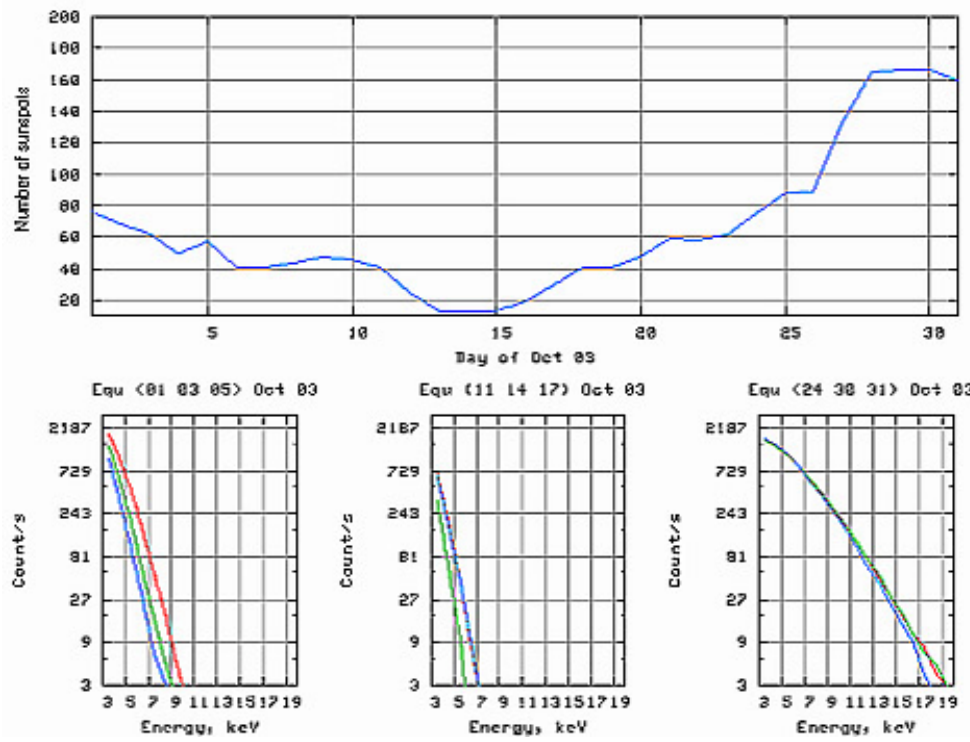

Figure 4. Background spectra dependence from solar activity level

486) (Fig.4).These spectra give an idea of pre-flare heating and can be used to improve the forecast of solar flares.

The Flare Spectrometer IRIS is used to measure flare-generated X-rays $(2-200 \mathrm{keV})$ with a high time resolution $(10 \mathrm{~ms})$. Measurements are made simultaneously in several energy channels. The most direct evidence of magnetic reconnection processes in solar flares is a fast time structure of hard X-rays. Sub- second pulses detected in nonthermal hard X-rays are generally interpreted as bremsstrahlung from electrons accelerated in the lower corona. The "IRIS" scintillator detectors record hard X-rays in $20200 \mathrm{keV}$ in four energy channels with the time resolution of $10.4 \mathrm{~ms}$ (flare mode). The time profiles of the hard X-rays detected in the solar flare of August 20, 2002 displayed an outstanding feature of this flare - a fast burst with duration of $80 \mathrm{~ms}$ and FWHM about $30 \mathrm{~ms}$ detected at 28:16.77 UT (Fig.5). The confidence level of this peak is 6 ? and the time profile includes 8 time points following each other at $10.4 \mathrm{~ms}$ intervals. These facts exclude accidental nature of this pulse. The same time structure was not observed in the rise and peak phases of the flare. 


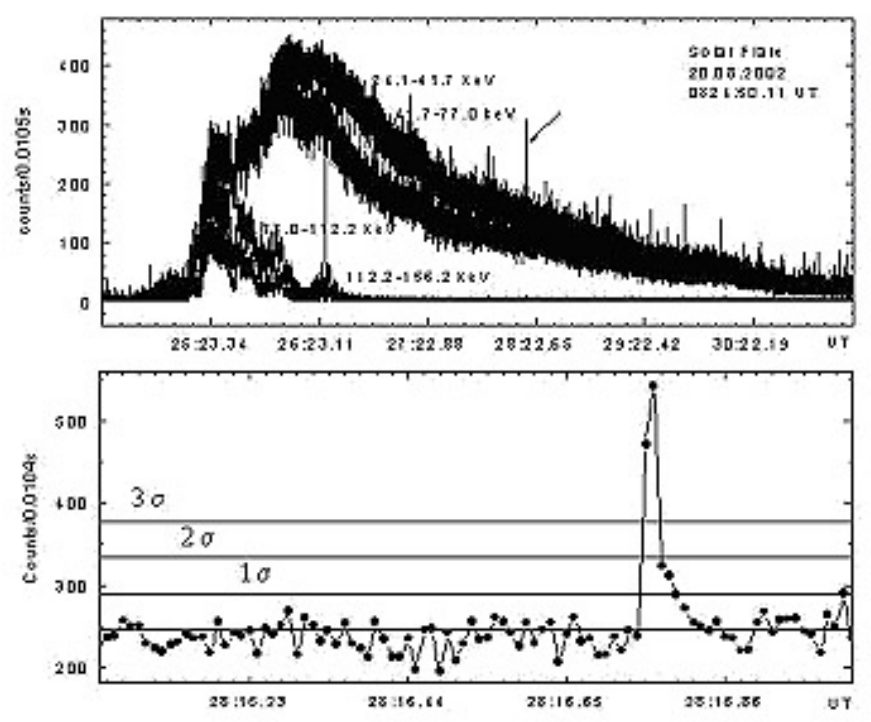

Figure 5. The time profiles of hard X-rays detected in solar flare of August 20, 2002.

DIOGENESS Spectrophotometer and X-Ray Spectrometer RESIK operating in the 3-7 A X-ray spectral range have detected multiple spectral lines in the emission of the most intensive solar flares ever observed. DIOGENESS has observed for the first time the evolution of spectra in vicinity of He-like ions of Si XIII, S XV and Ca XIX He-like ions with high resolution. This has been possible for the entire duration of the X5.3 class flare of 25 August 2001. Thanks of a special arrangement of the scanning crystals in so-called X-ray Dopplerometer configuration, absolute blue-shifts of entire emission lines have been observed allowing for estimates of absolute value of the hot plasma radial velocity component (Fig.6).

X-Ray Spectrometer RESIK has measured hundreds of thousands of spectra in the unexplored range between 3.3 A and 6.1 A. Tens of new spectral lines has been identified by investigating the variations of spectral shapes emitted by plasma of different temperature. Thanks to the high sensitivity of the instrument, week lines from $\mathrm{K}$ and $\mathrm{Cl}$ have been observed and absolute abundances of these elements in the corona have been determined for the first time (Fig.7).

The Solar X-Ray Spectropolarimeter SPR is measuring polarization of the solar-flare hard X-rays in the range of 20-100 keV, which occurs as high-energy electron beams interact with the dense solar atmosphere. A large polarization was observed during the bursts of emission associated with the flare of October 20, 2003, the harder radiation being stronger polarized. Then, a smooth transition to the background values took place by the end of the flare. At the beginning of the flare the polarization was $50-60 \%$ in the 20-40 keV channel and 70-100\% in the 40-60 keV and $60-100 \mathrm{keV}$ channels. Then, the polarization in the $20-40 \mathrm{keV}$ channel was decreasing monotonically over the entire time profile of the flare. In the $40-60 \mathrm{keV}$ and $60-100 \mathrm{keV}$ channels, the degree of polarization was the greatest at the peaks of the flare intensity. In the flare of October 29, 2003, the polarization was in east-west direction.

The Gamma Spectrometer HELICON is measuring the flare-generated hard X-ray and gamma radiation with a high temporal and spectral resolution in eight energy channels. 

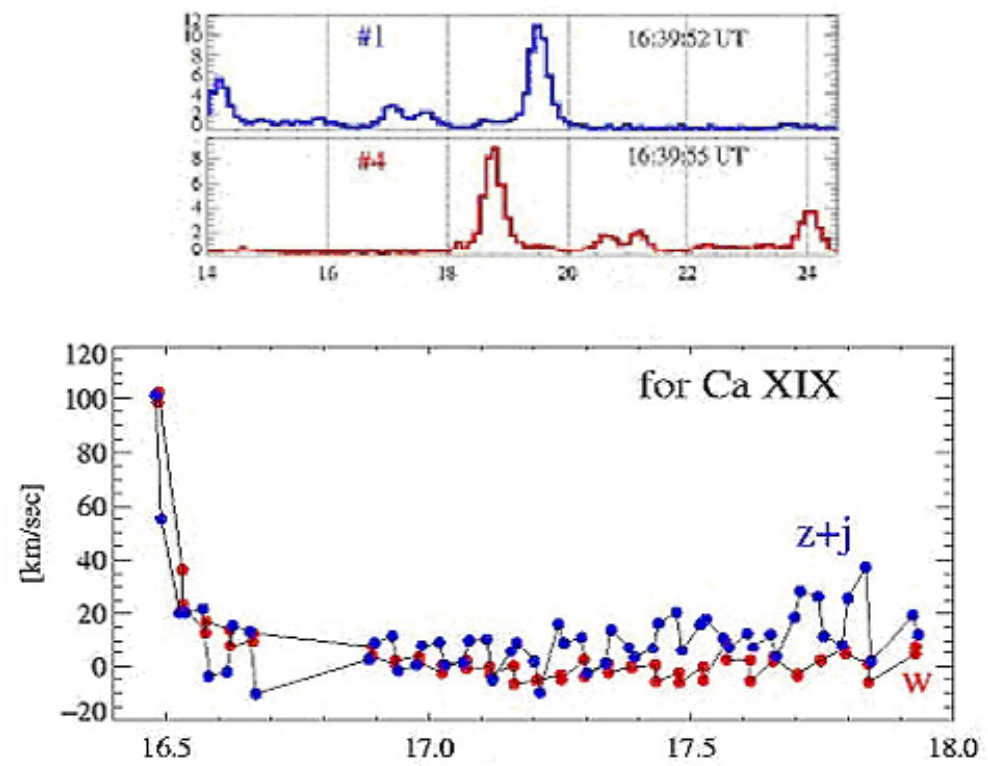

Figure 6. First absolute determinations of the Doppler shifts for X-ray lines formed in hot multimillion degrees solar flare plasmas - detection of fast plasma motions during early phase of 25 August 2001 X5.3 flare.
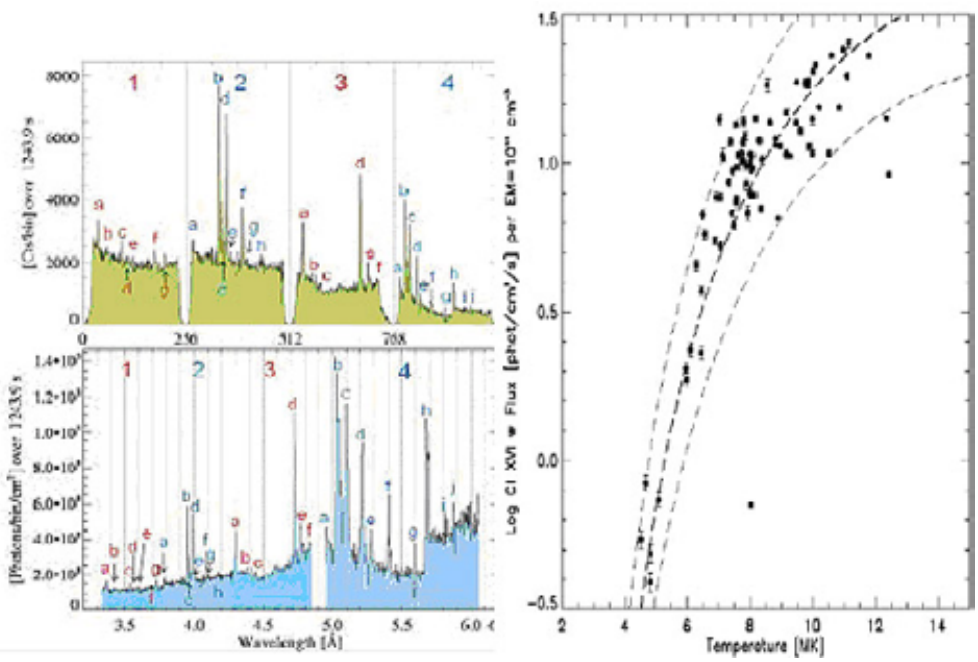

Figure 7. First absolute determinations of the potassium and chlorine abundances in the solar corona

On the basis of these measurements, the dynamics of the hard radiation spectrum has been studied in all phases of the flare, including the characteristic values and time-scales of variations in the spectrum inclination.

More than 30 solar flares of class M2 and higher in GOES classification were recorded during 2.5 years of AVS-F device (Amplitude-Temporal Spectrometry) operation in the energy range $E_{\gamma} \geqslant 50 \mathrm{keV}$. As it is well known, 30 active regions were present on the Sun during October-November 2003 according to SOHO data. Four regions (0484, 

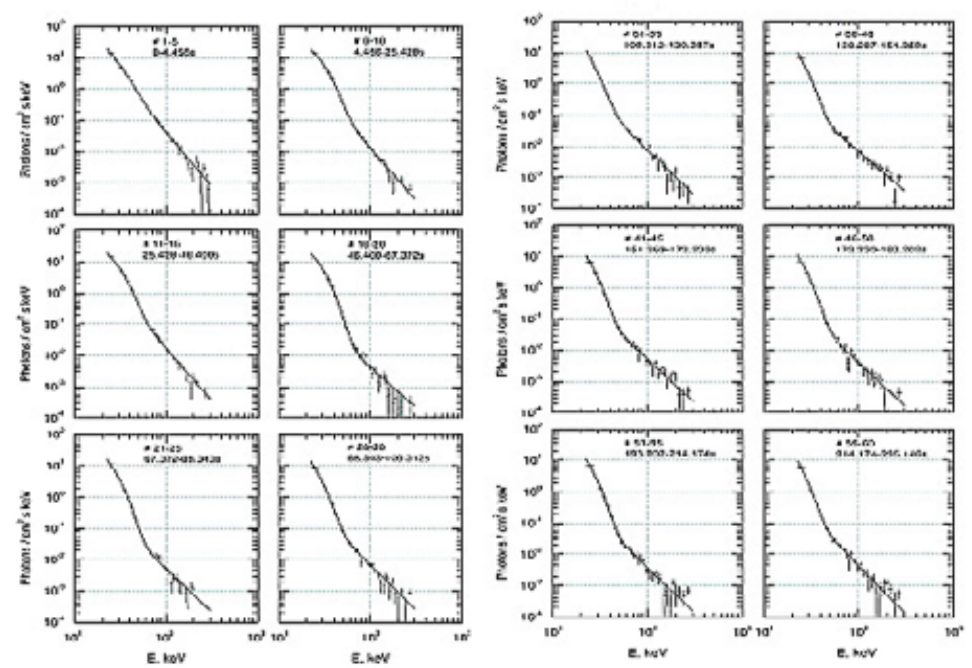

Figure 8. Successive energy spectra of the major flare of December 24, 2001, obtained in different phases of the event $(0-235 \mathrm{~s})$

0486, 0488, 0490) produced hard X-ray and gamma- ray emission. 14 flares of class M and $\mathrm{X}$ were detected during this time by GOES, HESSI and other experiments. Five solar flares were detected by AVS-F instrument at that time. One of the most intense events was observed on October 29 from 20:37 to 21:01 UT by the devices onboard GOES-12 (class X10, ball 2B, NOAA region 0486, S15W02). This flare was observed by AVS-F instrument at 20:39:00- 20:58:00 UT as the satellite was passing through the equatorial zone. Six gamma-line complexes were observed in the energy spectrum of this flare: $0.81-0.94,1.51-1.74,2.9-3.4,4.0-5.0,5.3-6.9 \mathrm{MeV}$ corresponding to ${ }^{56} \mathrm{Fe},{ }^{24} \mathrm{Mg}+{ }^{20} \mathrm{Ne}+{ }^{28} \mathrm{Si},{ }^{20} \mathrm{Ne}+16 \mathrm{O},{ }^{12} \mathrm{C}$ and ${ }^{16} \mathrm{O}$ lines, respectively. These lines and the line from the neutron capture at $2.2 \mathrm{MeV}$ were seen during $\sim 20 \mathrm{~min}$. The temporal profiles of the radiation in the energy ranges corresponding to prompt gammalines differ from that in the neutron capture line. Several temporal maxima were observed in all mentioned energy ranges. The maximum of emissivity in the gamma-lines does not coincide with the soft X-ray maximum observed at 20:49:00 by GOES-12. But one of the gamma-line emission maxima at 20:48 (in the energy ranges $0.81-0.94 \mathrm{MeV}, 2.14-2.64$ $\mathrm{MeV}, 4.0-5.0 \mathrm{MeV}$ and 5.3-6.9 MeV) coincides with the maximum detected in $12-25 \mathrm{keV}$ by RHESSI.

Measuring solar cosmic rays along its orbit with the SCR Complex (Gamma Emission Spectrometer SONG, Cosmic Ray Monitor MKL, Cosmic Emission Spectrometer SKI-3), CORONAS-F performs monitoring of radiation conditions in the near-Earth space and dynamics of the Earth's magnetosphere and radiation belts during active events in the Sun. The data obtained are used to study geomagnetic storms, penetration of energetic particles into the Earth's magnetosphere, and strong deformations of the magnetosphere and radiation belts. It is established that only $20 \%$ of the recorded flares have caused geomagnetic storms. One third of the flares were accompanied by the appearance of solar cosmic rays at the Earth's orbit. Gamma rays with the energy up to $100 \mathrm{MeV}$ and neutrons with the energy of hundreds of $\mathrm{MeV}$ were recorded simultaneously during the flare of August 25, 2001. 


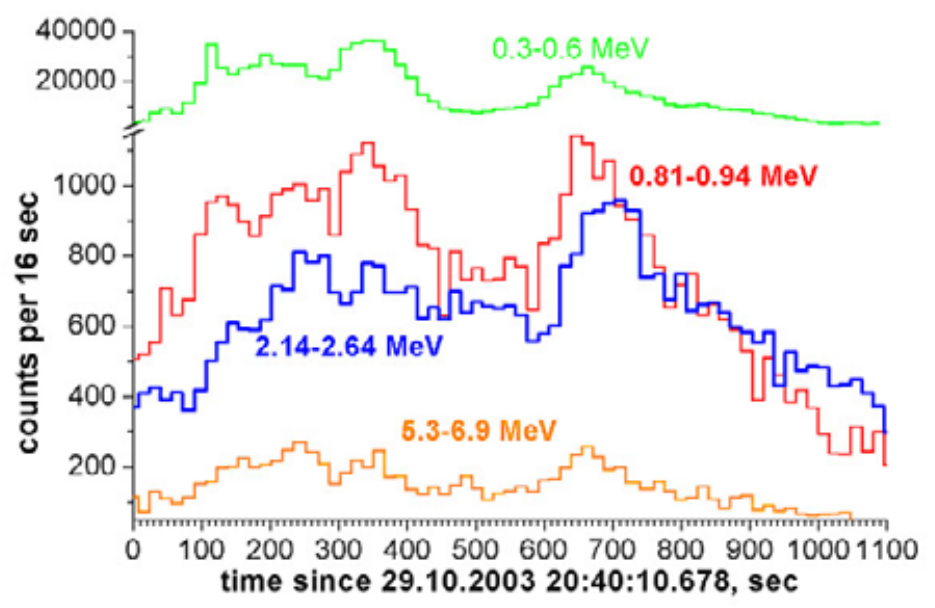

Figure 9. Temporal profiles of solar flare 29.10.2003 registered by AVS-F in the energy bands 0.3-0.6 MeV, 0.81-0.94 MeV, 2.14-2.64 MeV and 5.3-6.9 MeV

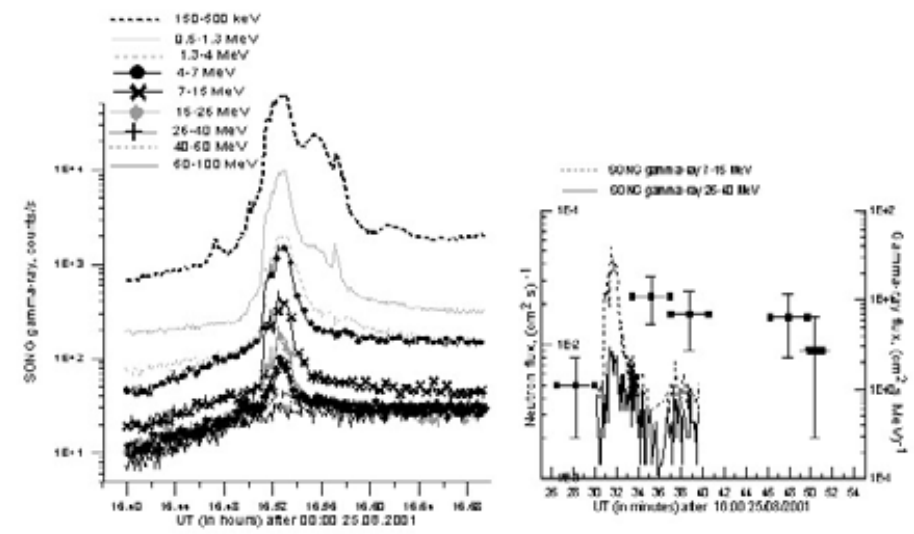

Figure 10. Gamma emission (up to $100 \mathrm{MeV}$ ) and neutrons (hundreds $\mathrm{MeV}$ ) for solar flare 25 August 2001

A large volume of data on the solar UV fluxes, which affect the Earth's upper atmosphere and serve as an important characteristic of solar activity during a cycle, have been obtained with the Solar UV Radiometer SUFR-Sp-K and Solar UV Spectrophotometer VUSS-L. It is found out that, even in the major flares, the increase of UV radiation at $120 \mathrm{~nm}$ does not exceed a few percent. The correlation between the solar UV and radio fluxes has been determined for different observations periods. It is used to estimate the UV radiation and monitor the Earth's atmospheric conditions in the periods when the satellite UV data are absent and the ground-based radio flux measurements alone are available.

Global oscillations of the Sun have been observed with the DIFOS Spectrophotometer in a broad wavelength range of 350-1500 nm, and new experimental data on their manifestations in the observed radiation have been obtained, namely a significant growth of the oscillation amplitude in the UV spectral range. It is established that the amplitude 


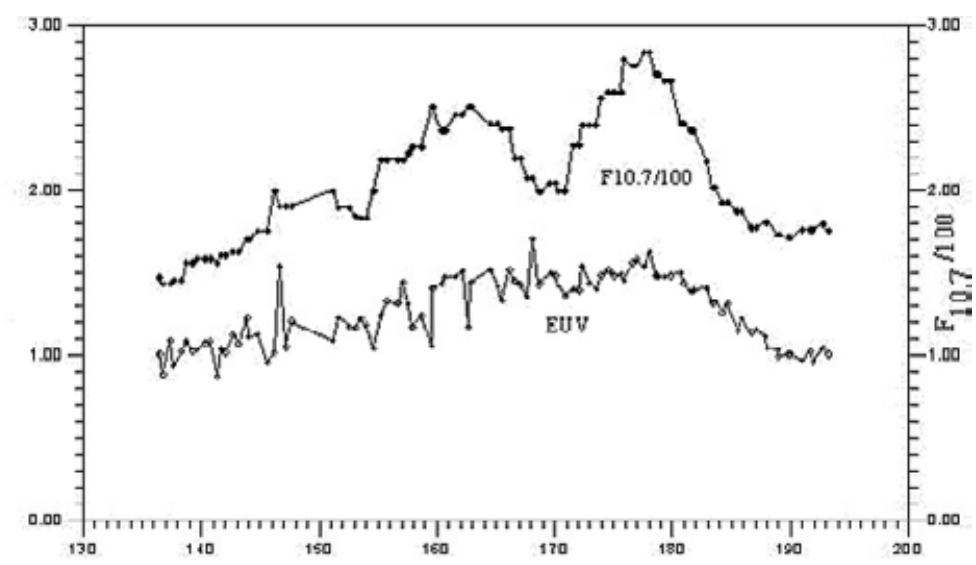

Figure 11. UV/Radio Flux Correlation

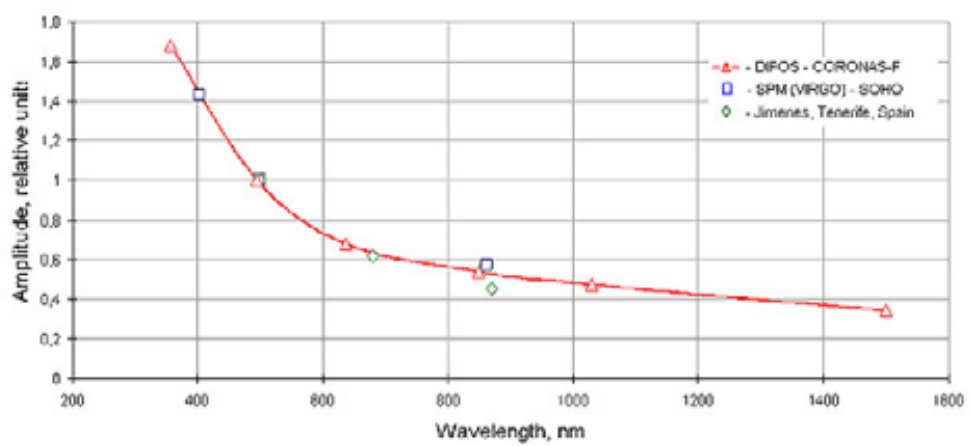

Figure 12. Relative amplitude of the solar p-modes versus observation wavelength

depends on the wavelength that agrees fairly well with earlier observations in more narrow spectral ranges.

For the entire period of operation, the satellite has provided more than a million solar X-ray spectra, more that 500 thousand high-resolution X-ray images of the Sun, numerous time profiles of the solar flare radiation in a broad energy range, new data on the solar cosmic ray and UV fluxes.

More early results from CORONAS-F satellite can be found in papers by Oraevsky et.al., 2002 and Oraevsky et.al., 2003.

\section{Acknowledgements}

We are grateful to CORONAS-F team for having placed at our disposal the processed data, which are used in this paper.

\section{References}

V.N.Oraevskii, I.I.Sobelman, I.A.Zitnik, V.D.Kuznetsov, Comprehensive solar studies by CORONAS-F satellite: new results., Physics Uspekhi v.45, N 8, 2002, p.886-896, 2002.

V.N.Oraevskii, I.I.Sobelman, I.A.Zitnik, V.D.Kuznetsov,A.I.Stepanov, G.M.Polischuk, P.N.Kovilin, A.A.Negoda, V.I.Dranovsky, Ya.S.Yatskiv, CORONAS-F observations of active phenomena on the Sun., Adv. Space Res., Vol. 32, No.12, pp.2567-2572, 2003. 